\title{
Teaching of optics to mechanical engineering students
}

\author{
Fromund Hock
}

Fromund Hock, "Teaching of optics to mechanical engineering students,"

Proc. SPIE 1603, Education in Optics, (1 March 1992); doi: 10.1117/12.57896 


\author{
Fromund Hock \\ University of Hannover, FRG, \\ Department of Mechanical Engineering \\ Institute of Engineering Metrology
}

\author{
Teaching of optics to \\ mechanical Engineering Students
}

The profession of the mechanical engineers leads to different, but systems related occupations of the engineers as design, development, production systems, integrating, systems operation, systems maintenance, materials science, control science and last not least the quality control from the design quality, the quality of production systems and the products quality and last not least to the quality influence of techniques to the biosphere.

Most mechanical engineering students are not minded to abstract imaterial things like rays, waves, intensity and photon energy distributions of radiation in space and time, things they cannot take into their hands.

But the proper operation of systems changed in the last decades from the dependence on mechanical structures like guide ways, gears etc. that means shapes and dimensions of interacting parts, to the systems stabilisation by control loops, guiding energy, temperature, pressure, position, speed, mass flow, volume flow, radiation as a tool, etc..

The function of technical systems depends more and more on gathering, transferring, processing and storing of informations. one important goal of advanced techniques are self learning systems. CIM is a step in this direction.

The intention to use flexible systems in production, transport etc. drives to the application of flexible control loops, structured by the software.

With the increasing throughput of material, energy and information through a technical system the human operator is not only overstressed in sensor and control functions but also in the recognition of acoustical or optical patterns.

The education of mechanical engineering students in optics is aside of the very rudimentary education in physics aside of electrotechniques and thermodynamics mainly a part of the education in measurement and control. It is not advisable to direct this education to the state of the art of instrumentation for measurement and control. Compared to the professional life span of an engineer the changes in the realisation of measurement and control instrumentation are so fast that the ability for life long adaptive learning must be the most important goal of education in engineering. 
So I have not to repeat the intentions of Adolf Lohmann to concentrate to the basics. But the presentation of the basics to the practical minded students needs always an examplification, for what good.

In the relations between the institutes in an engineering department you mostly have the tensian between the big applied institutes, research orientated and the institutes loaded more by performing the basic education. The applied institutes demand students trained for their actuell measurement and control instrumentation but don't like to loose their fractions in the time budget of the students to a higher fraction in the education for measurement and control.

From the general systems view the needs of collecting and transferring information have to be estimated in terms of speed, sampling frequency, allowable error budget, that is basicly the channel capacity.

A student has first to estimate what physical quality gives, by which signal parameters, an optimal adaption to the information needs of the system under investigation. Under these aspects they have to know that optical radiation could be the fastest information carrier with the broadest range of signal parameters with the possibility to operate a lot of information channels in parallel. This basic knowledge has to be supported by examples using different optical signal parameters as radiated flux, optical frequency, intensity modulation frequency, phase relation between two beams, polarisation, lateral or angular beam position etc., for different tasks in connection with different light sources and receivers.

Examples of topics:

Thermal sources, flourescent sources, lasers, light amplification in diodes and fibers, the photographic emulsion, the different photoelectric thermal and quantum detectors as current voltage and noise sources and their electrical circuits, influencing the bandwidth of the measuring signals. The geometrical and wave optics can be only correlated to a small number of typs of optical instruments from telescopes, microscopes, optical comparators, laser scanners and triangulation distance sensors to interferometers. 
In the layout of microscopes, simple cameras, telescopes and optical comparators the position of the projection center of the images, as far as possible telezentric ray traycing, is important for errors in scale factors. The ruling function of the sin. law for the size and used aperture of the light source to object field and size and aperture of the optical receiver is another point of view, related to resolution in lateral and axial direction and to the radiation flux available at the receiver.

As an example in some more depth, the coherent optics is introduced by the two beam interferometers, important to length, surface and vibration measurement. Starting with the Twaiman green design, (Fig. 1) well known to the auditorium. All two beam interferometers can be generalized to the combination of two identical light sources, generated by the not shown beam splitter, together with their identical collimatorlenses, united by the not shown beam combiner and observed by a telescope lens, projecting fringes of equal inclination into the focal plane or enabling the observation of ringes of equal thickness through an restricted homophase area of the focal plane (Fig. 2).

The angular and distance relations of these three basic components in space give access to the fringe positions, the interference contrast and to their shape and in this way to the measurement errors due to the interferometer geometry. From this point of view the relations to Abbee's rule on first and second order length measurement errors can be introduced. We have to look for 4 directions in the geometry of length measuring instruments (Fig. 3). In the interferometry to the direction of the illuminating beam, the direction of minimum path difference in the interferometer, the direction of the commun movement of the measuring reflector (triple prism, cats eye) and the object under measurement and the direction of the length to be measured. Taking cats eyes as tilt insensitive reflectors instead of plain mirrors in a length measuring interferometer leds directly to the interference microscope by observing the mirrors in the focal plane.

Phase shifting techniques by geometrical or physical phase retarders have to be introduced for up-down counting techniques and are related to strainoptics (Fig. 4).

By regarding a linear or radial grating as an interferometric beamsplitter, length and angular measurement by metrological gratings can be related to two beam interferometry and enables the student to understand the use of phase gratings as length and angular standard (Fig. 5). 
The phase shifting techniques for up-down counting and fractional fringe measurement are basicly the same as in wave length related interferometry. The achromatism is gained by a grating as beam combiner (Fig 6.).

By changing from a beam combining grating in length measurement to a beam combining single scatter partical, there is an direct access to fluid flow measurement by achromatic Doppler anemometry (LDA) (Fig. 7).

In the cases of wavelength or grating pitch related length or angle measurement or the LDA measurement the phase and fringe number measuring electronics is to be shown together with the optics for their designs are interrelated for maximal signal quality (Fig. $3,4,8,9$ ).

From single and multi particle or rough surface scattering of coherent lights, the speckle phenomena can be introduced as statistical markers on surfaces under coherent illumination either for contactless speed or length measurement in production or the basic feature for double exposure photography and image evaluation by Young's fringes. From this knowledge there are paths to speckle photography, electronic speckle evaluation and the interference holography of technical objects.

Some additional time has to be devoted to guided light waves in multi- and monomode fibers and some basic integrated optic

structures.

A mechanical engineer who remembers to basic facts, methods and interrelations in optics and has not forgotten basics in signal processing might have access to optical instrumentation and measurement and control methods after some decades of professional life and permanent change of the realisations of the instrumentation techniques needed for the measurement of dimensions, cinematics (speed, flow, vibration), dynamics (mass, force, energy, efficency), thermodynamics (temperature, heat), process control (materials exchange, energy exchange, analyties, environment protection (emission, immission) or in biological or medical applications.

Another point of view for the education of mechanical engineers in optics is how to operate and maintain optics and optoelectronics in a hostile environment. This is a task for the education of engineers in institutes doing $R+D$ in applied engineering using fiber optics, window protection techniques, cleaning or cooling liquids as optical immersions, instrument cabinets, with inside overpressure etc. 
But the most important goal in all technical education is to combine basic and mental interrelated facts and methods knowledge with the motivation of life long learning and the motivation to the mental risk of combining not yet used or new effects and methods to invent and develop more advanced solutions for the needs of the human society saving environment and ressources.

Staying in a town of important changes, not only in the sovjet society, but for the whole world, I think we have to thank the Russian population having avoided a backslash in there social development and I suppose, returning to our congress that the progress in instruction and education in the Sovjet Union having teachers like Sacharow, was and is last not least the basis to avoid irrational decissions and actions in the future life of the populations in the republics of a sick world power. 


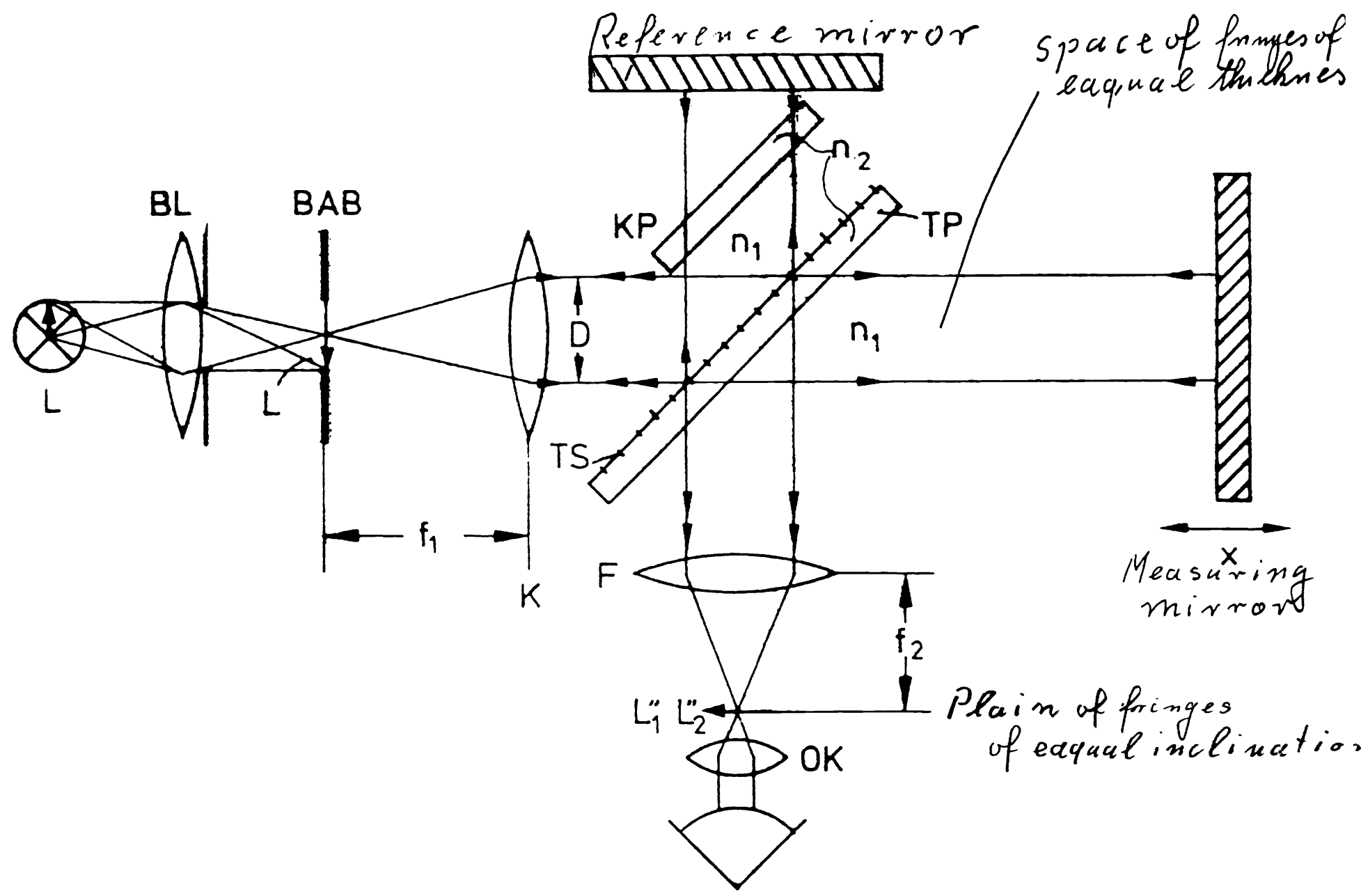

Fig. 1) Twyman Green interfeometer, observation of fringes of equal inclination and equal thickness

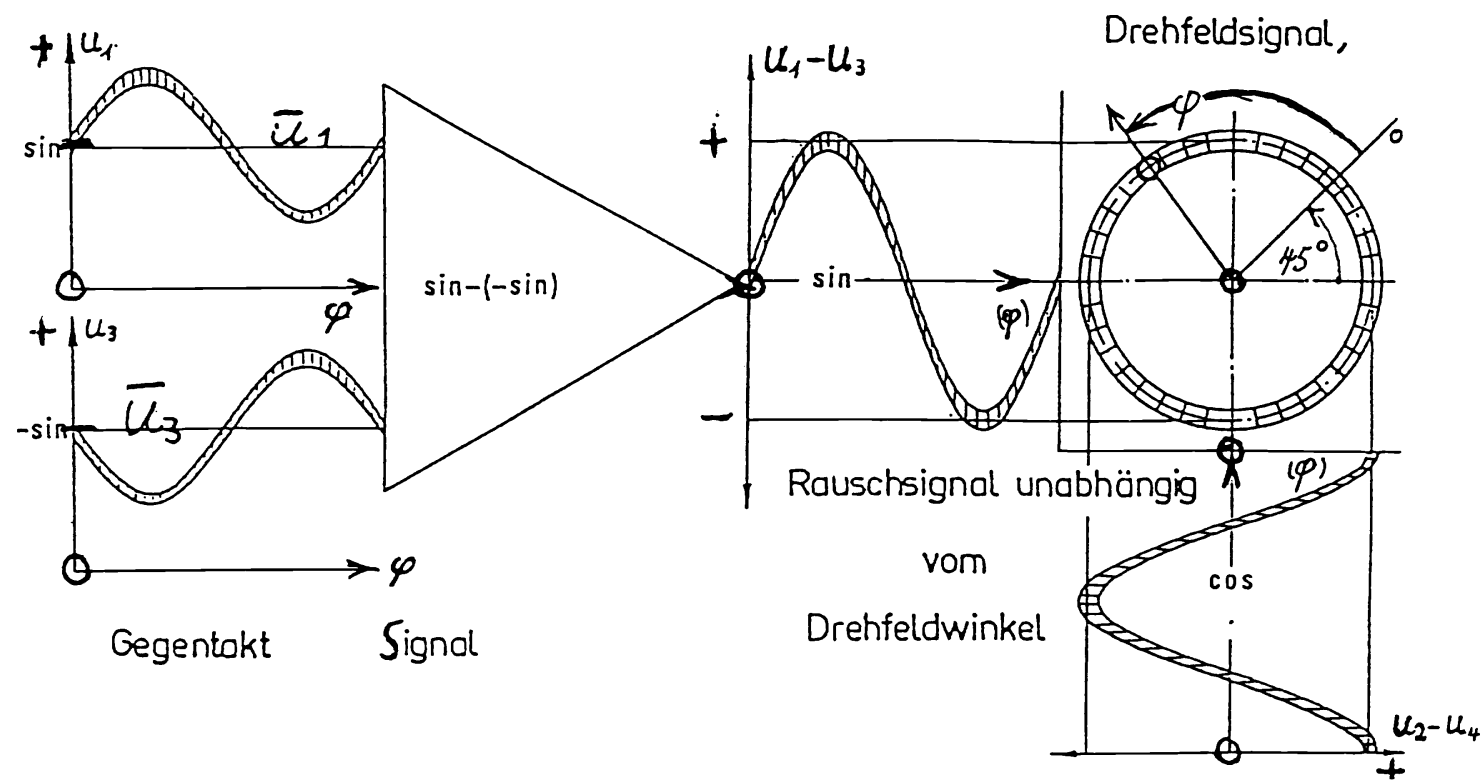

Fig. 4a) DC compensated quadratur signal pair to extract the measuring information by a rotating electrical pointer as phase angle modulo $2 \pi$ and the sign of rotation 


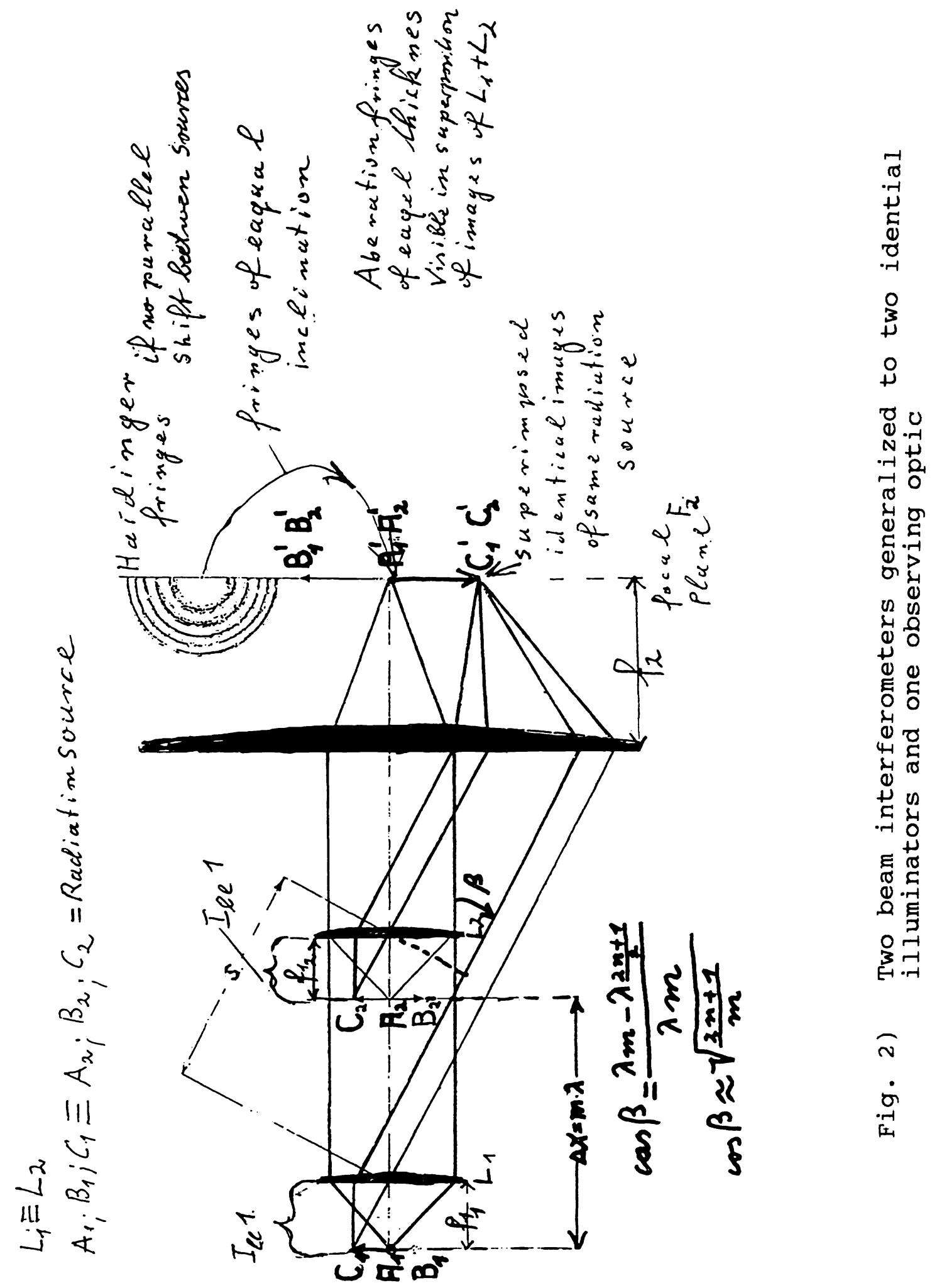




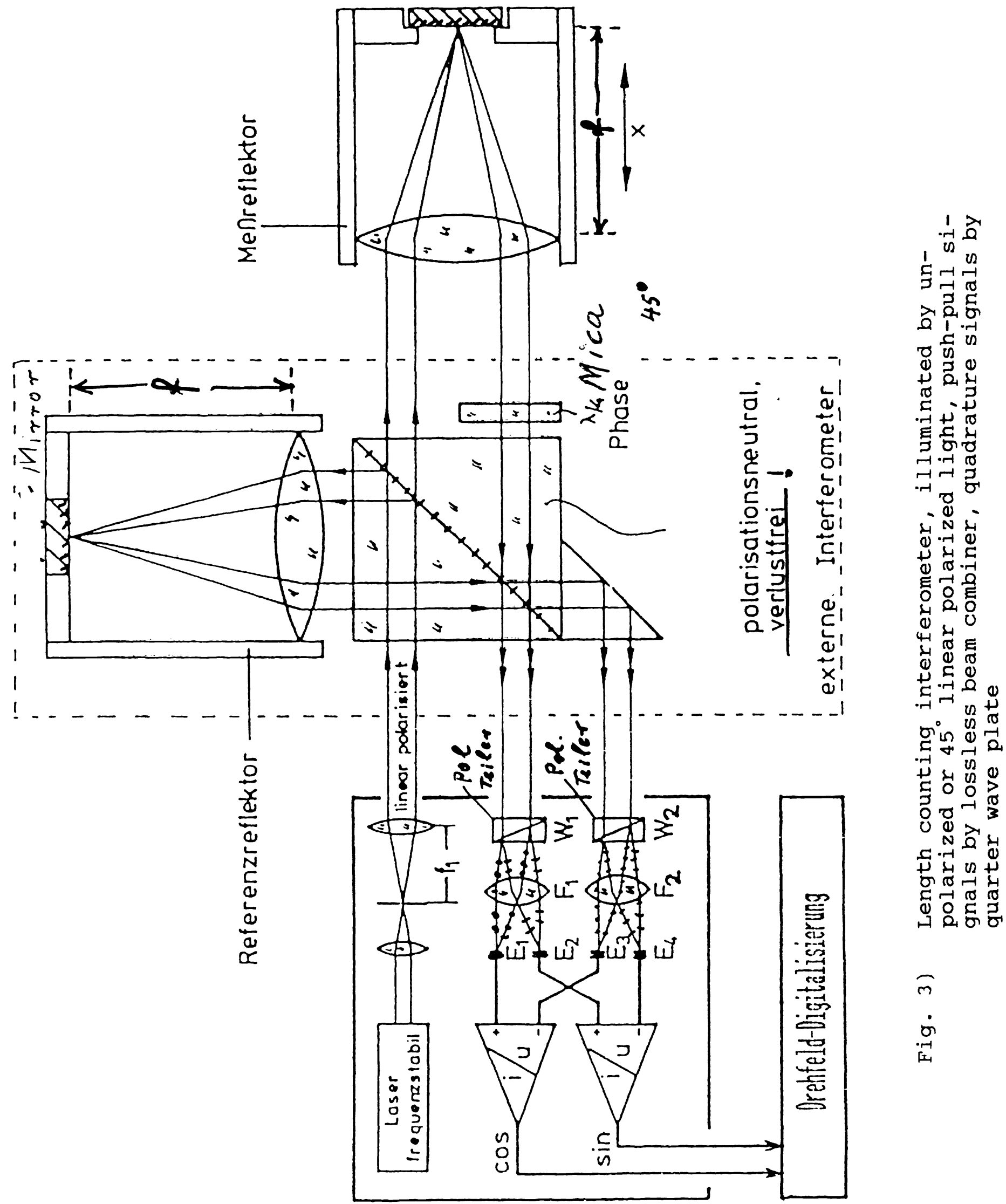



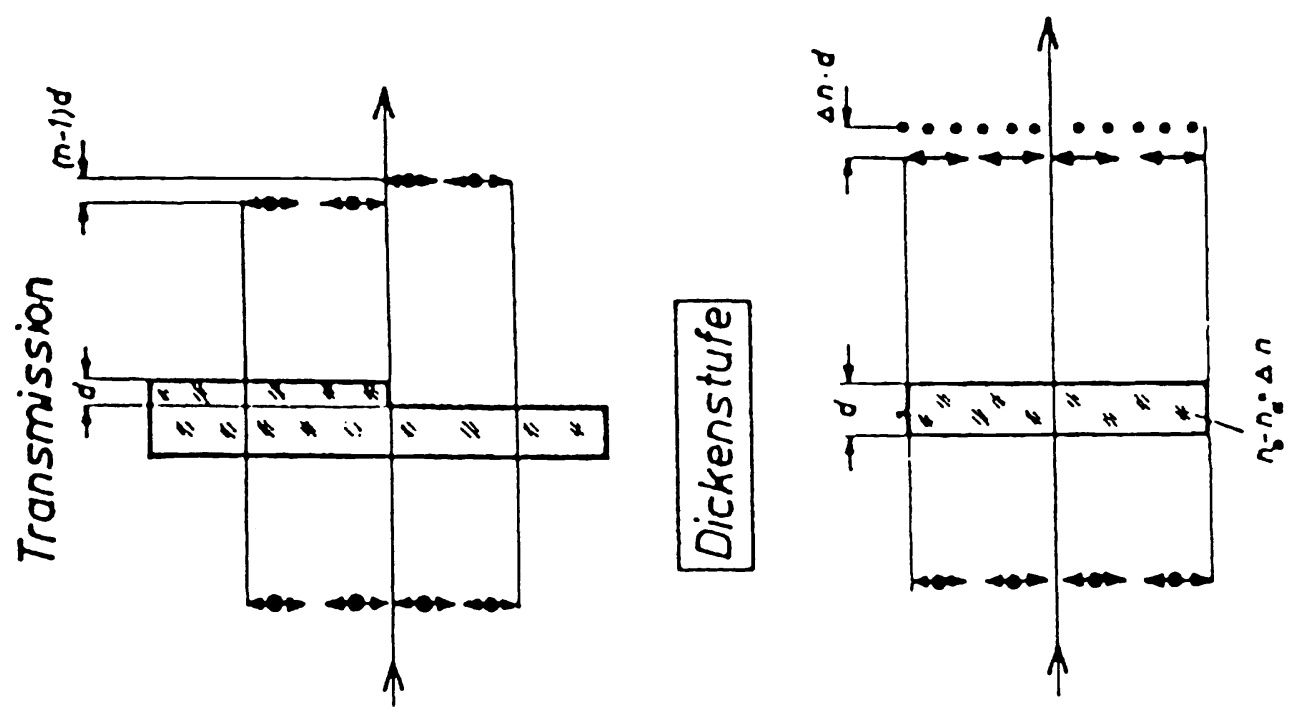

0
2
3
+1
0
0
0
0
2
0
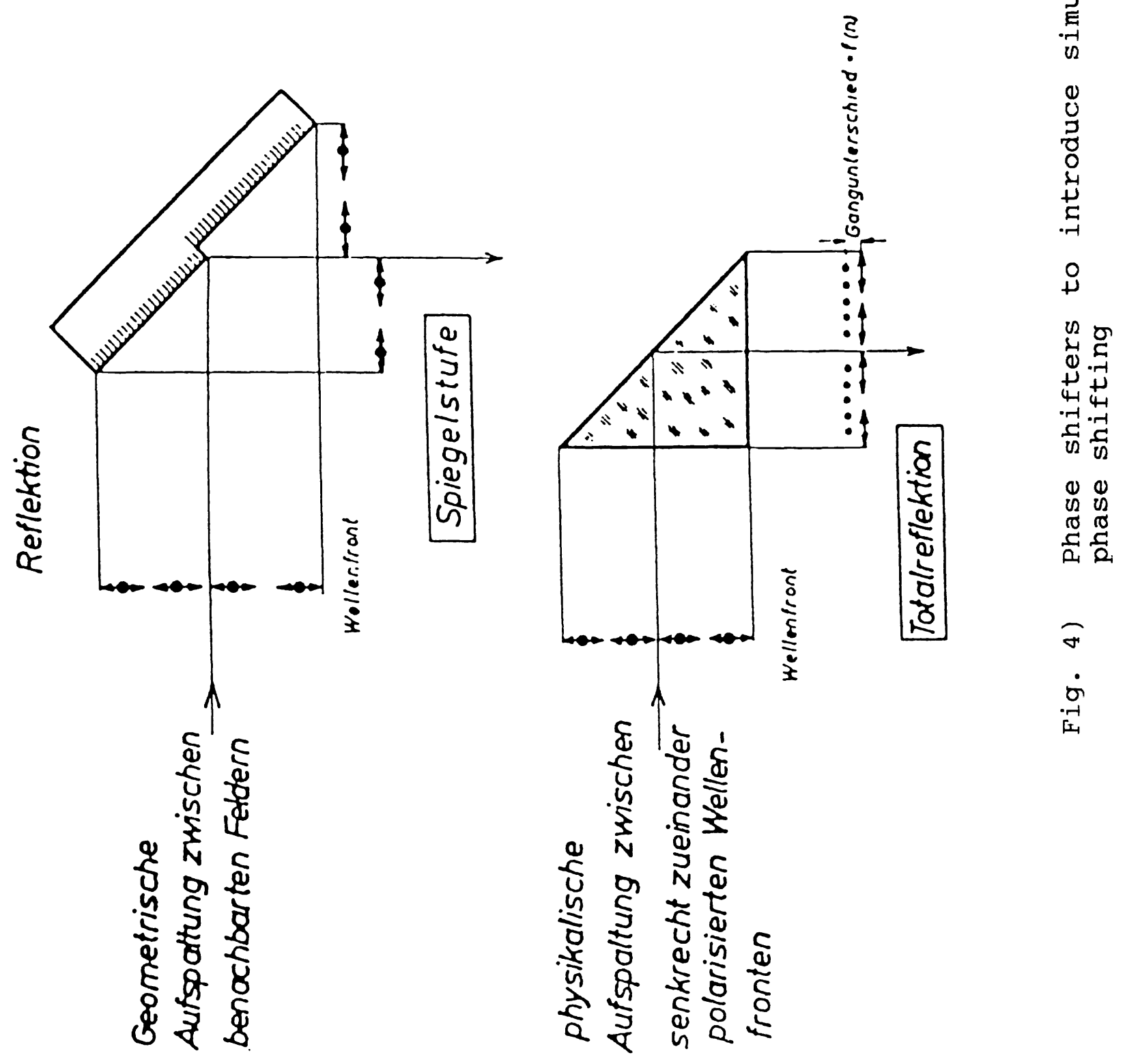


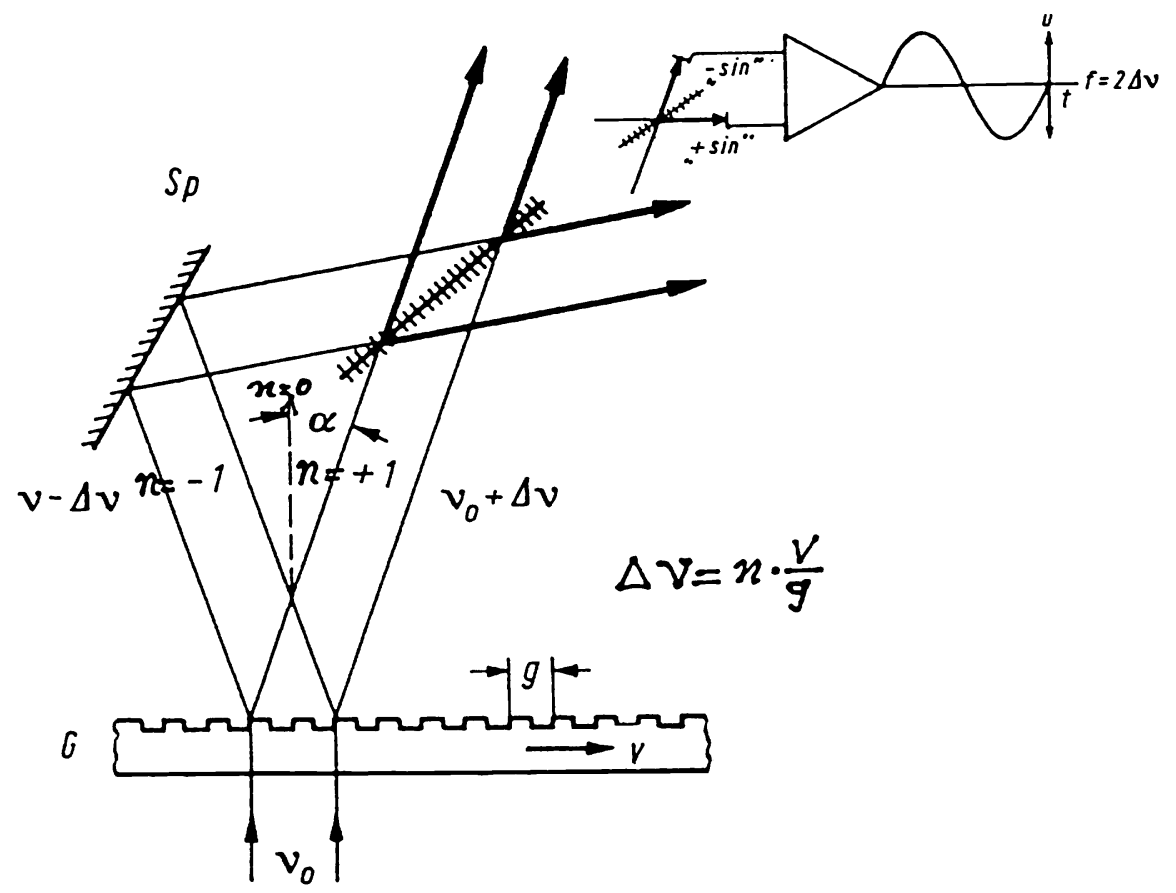

Fig. 5) Grating scale, signal generation by the simpliest possible two beam interferometer, beam splitter-grating beam combiner-lossless semitransparent mirror

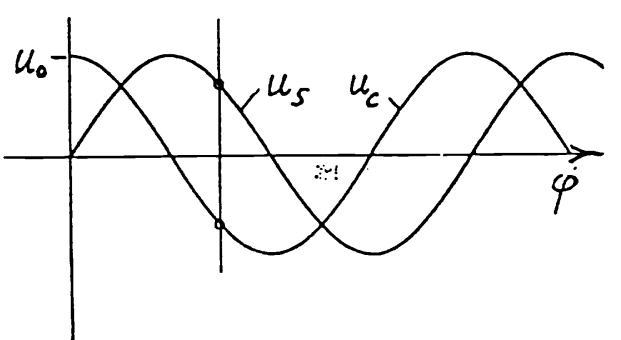

$\tan \varphi=\frac{u_{s}}{u_{c}}=\frac{R_{1}}{R_{2}}$ if $u_{D}=0$
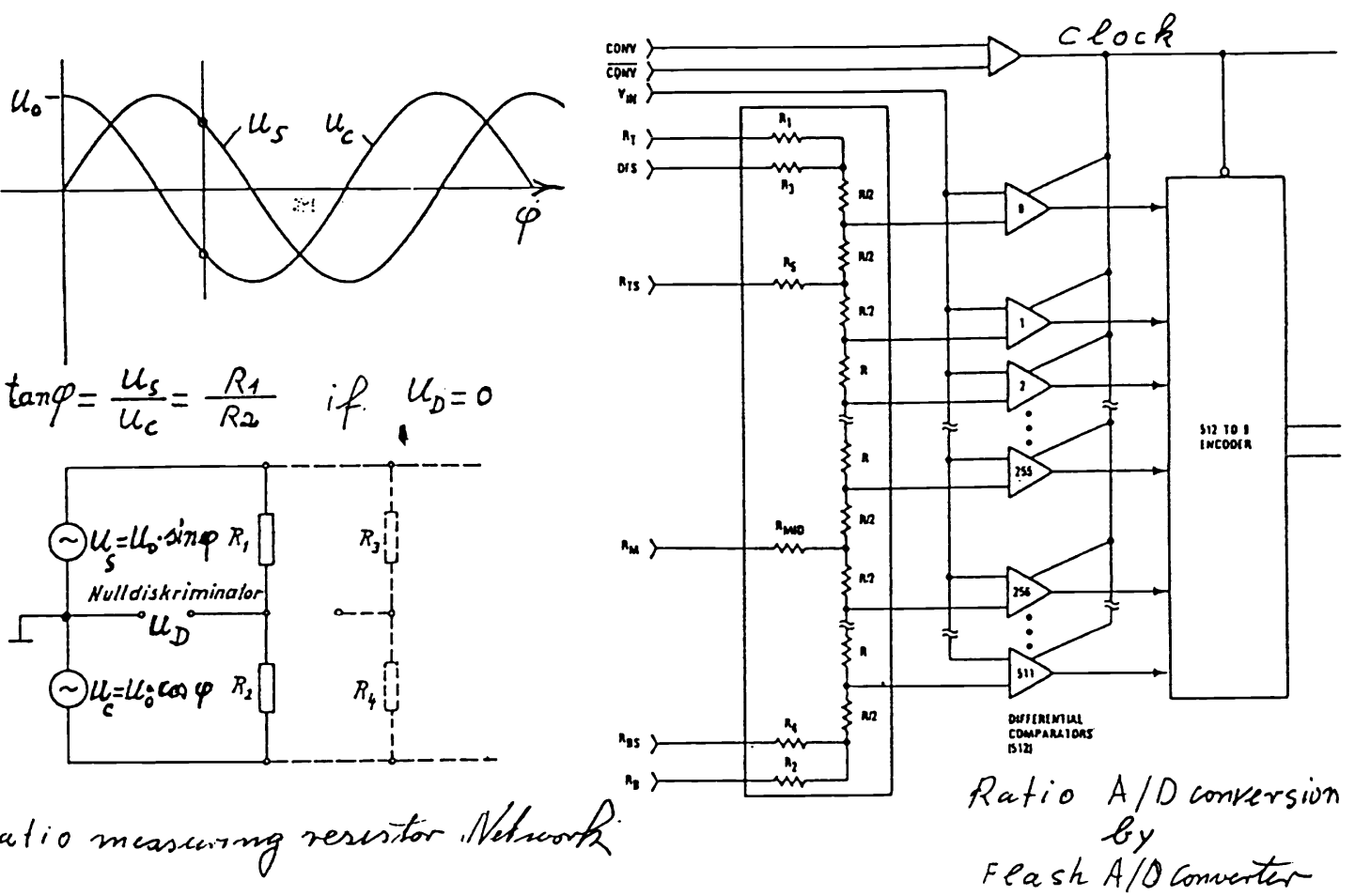

Fig. 8a) Digital measurement of quadratur signal electrical pointer, phase angle 


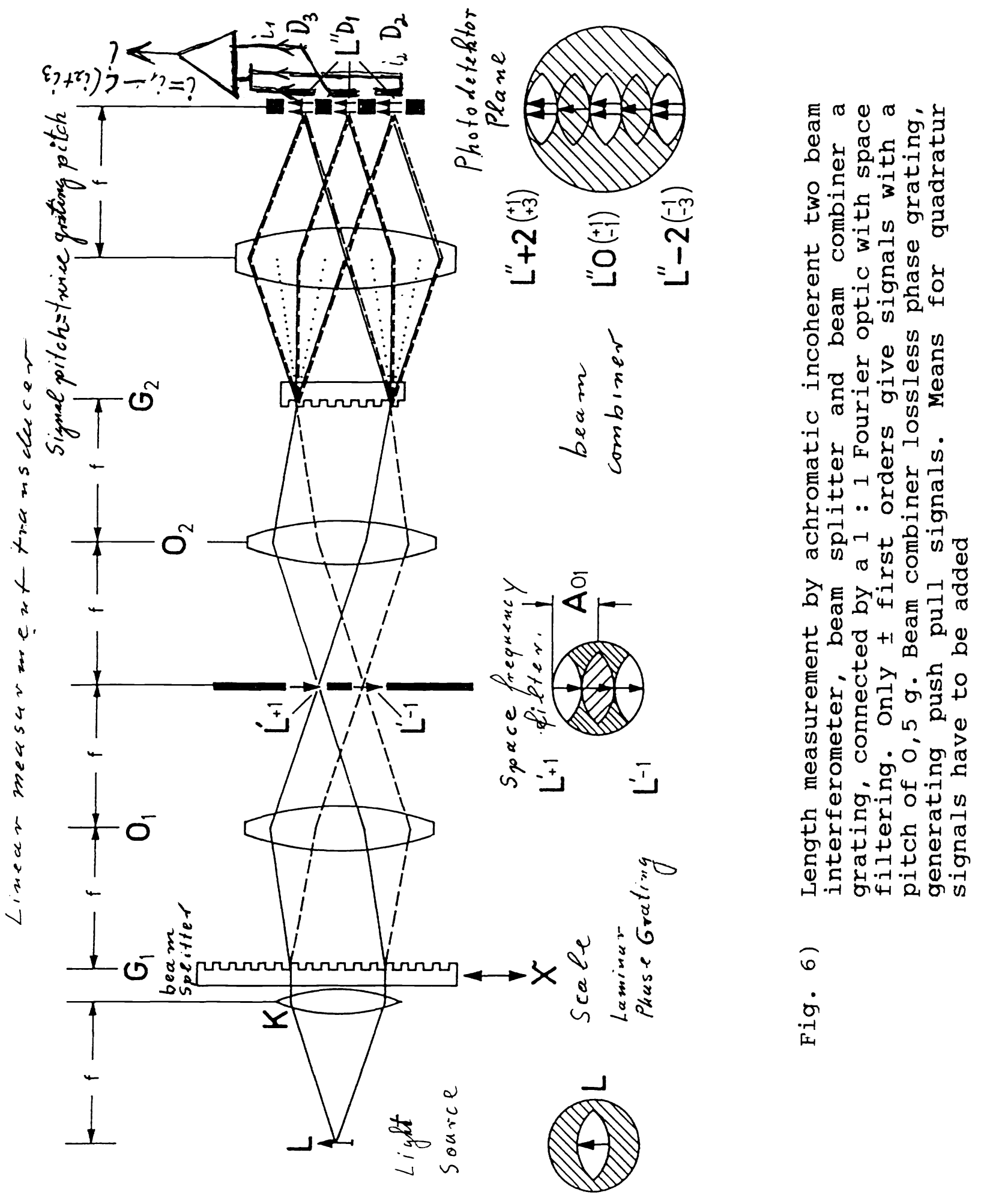




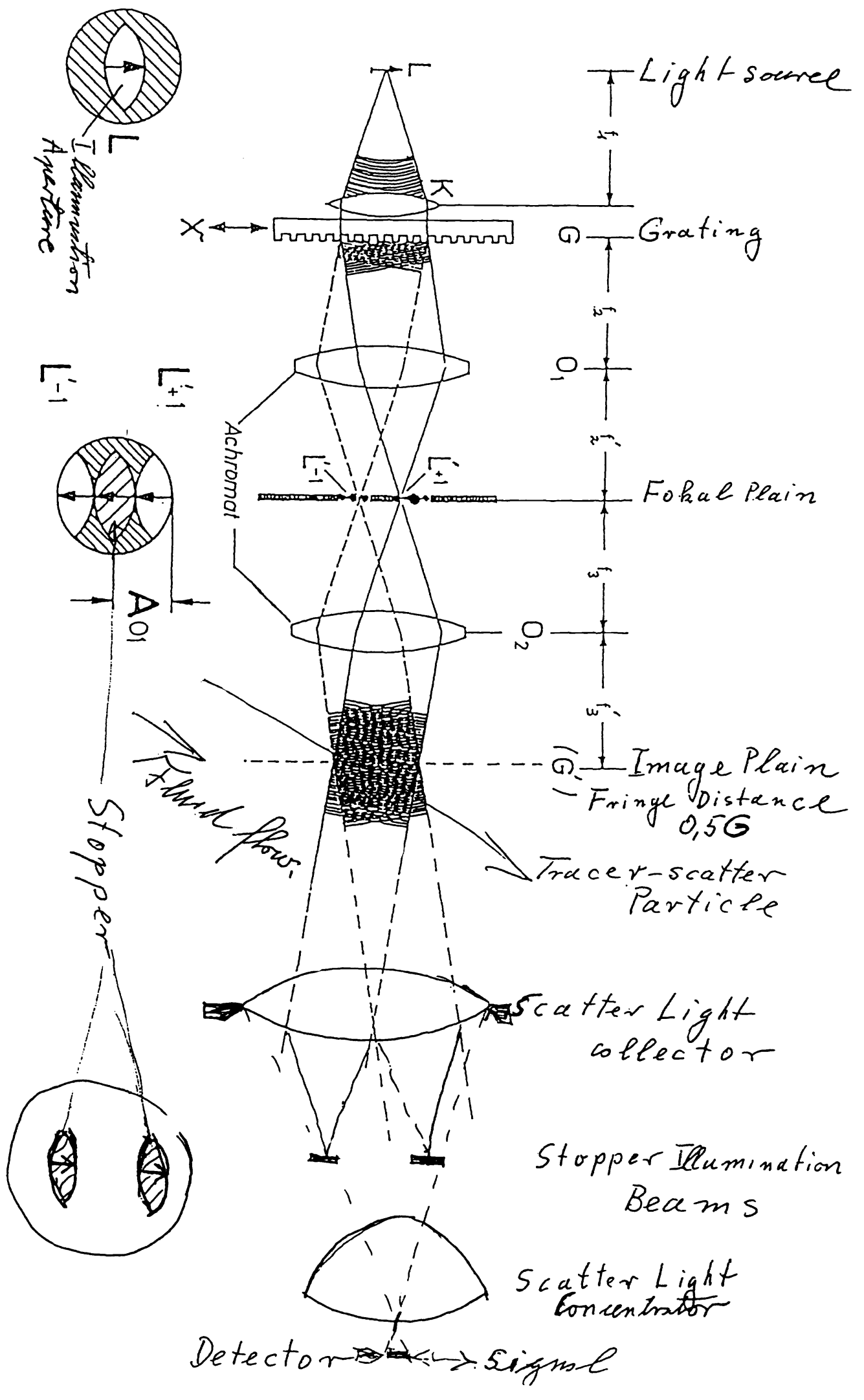

Fig. 7) Doppler anemometer, grating is beam splitter, scatter particle is beam combiner, achromatic compensated 


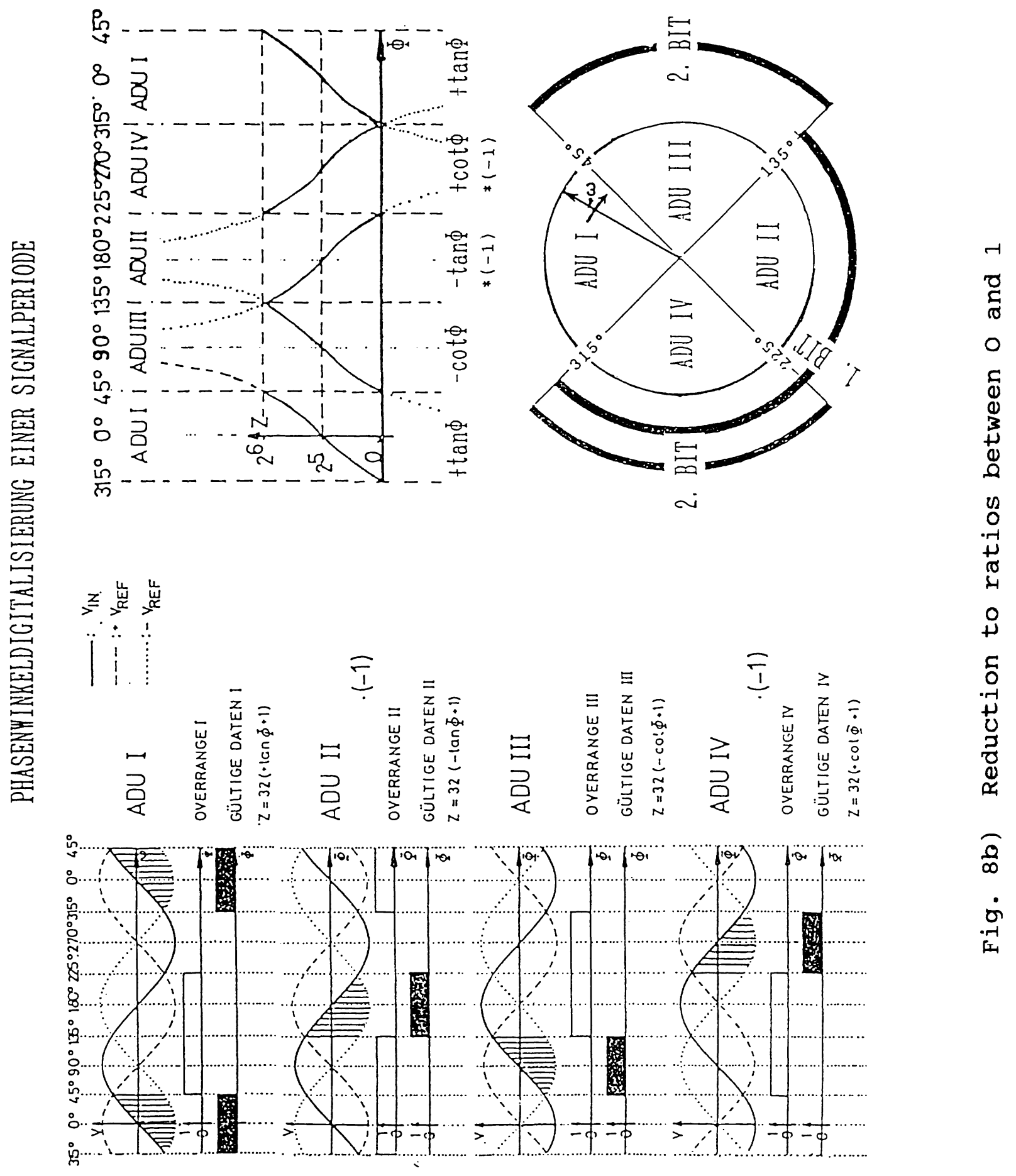




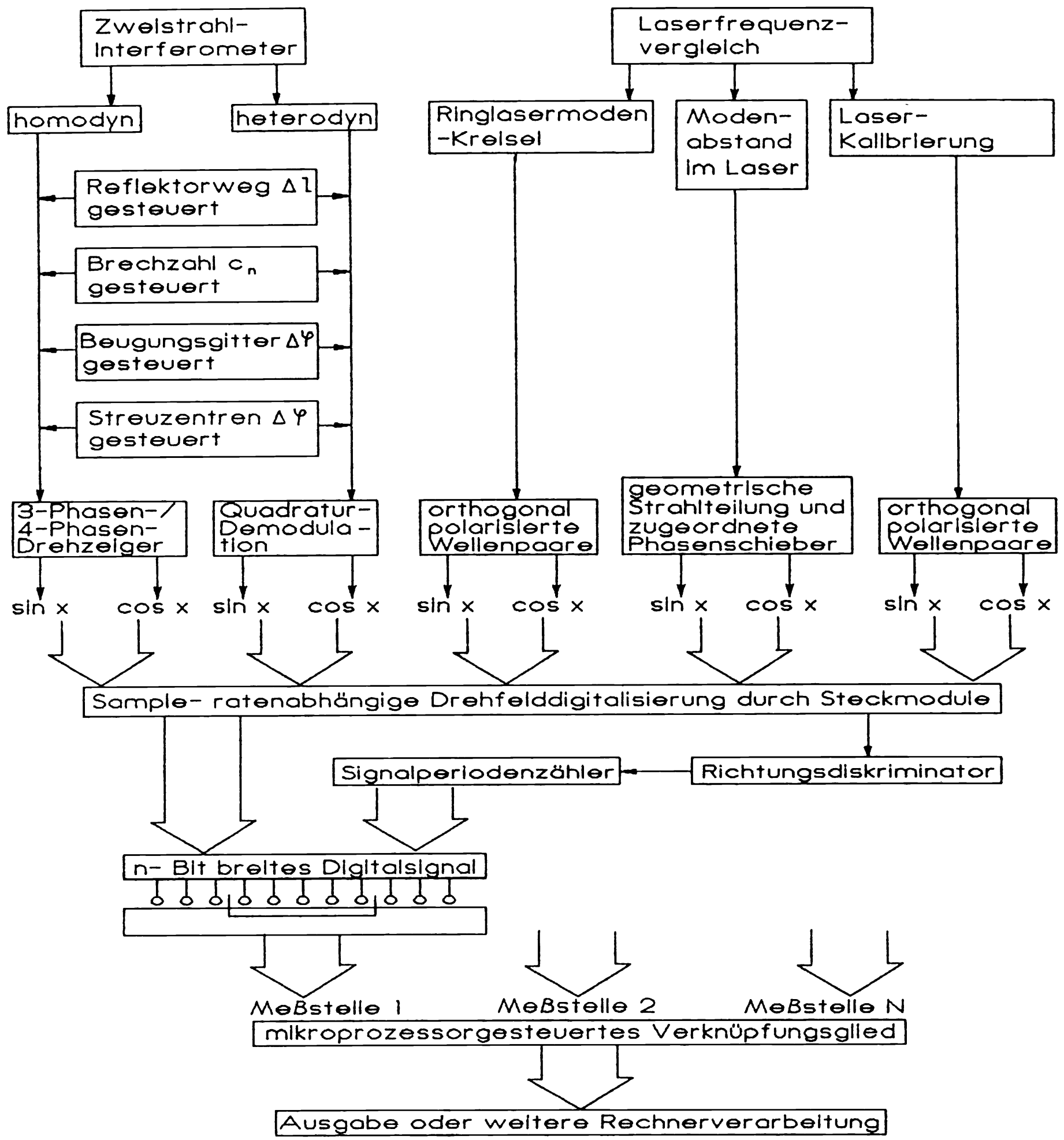

Fig. 9) Family of solutions for measuring tasks using the optical signal parameter phase 